\title{
Manuel M. Ponce en Sudamérica (1941)
}

\author{
por \\ Jorge Barrón Corvera \\ Universidad Autónoma de Zacatecas, México. \\ jorgebarron_corvera@yahoo.com.mx
}

Entre agosto y diciembre de 1941, Manuel M. Ponce (1882-1948) realizó una gira artística por Montevideo, Buenos Aires y Santiago de Chile. Presentó tres conferencias sobre música mexicana y cuatro conciertos con sus propias obras orquestales dirigidas por él mismo. Fue así el primero en llevar música sinfónica mexicana a esas capitales ${ }^{1}$. El repertorio incluyó Estampas nocturnas (para orquesta de cuerdas, ca. 1910), Concierto para piano (1911), Chapultepec (1922, revisado 1934), Pequeña suite en estilo antiguo (versión orquestal estrenada en 1935), Poema elegíaco (1919, revisado en 1934 y 1937), y los recién concluidos Ferial (1940) y Concierto para guitarra (1940). Sostuvo, además, diversas actividades de intercambio cultural. La gira fue un éxito y constituyó una elevada cima dentro de la trayectoria del compositor. La cobertura periodística fue tan copiosa como positiva. El punto focal fue el estreno mundial del Concierto del sur para guitarra y orquesta con la participación del guitarrista español Andrés Segovia, a quien está dedicada la obra. Opus de prolongada gestación, el Concierto representa el pináculo del prolífico catálogo guitarrístico de Ponce, mismo que constituye una aportación ineludible a la literatura universal de la guitarra.

Ponce cumplió cincuenta y nueve años de edad durante la gira (8 de diciembre de 1941). Estaba en plena madurez creativa y gozaba de merecido prestigio. Durante poco más de cuatro meses y con una apretada agenda, el artista disfruta significativos momentos. Triunfa como compositor, director y conferencista. Viaja en avión por primera vez. Vive emotivos encuentros con grandes personalidades. Francisco Curt Lange, Erich Kleiber, Aaron Copland, Nicolas Slonimsky y especialmente su viejo amigo Andrés Segovia, por mencionar sólo algunos. En México, sus amigos y colegas lo reciben con homenajes.

\section{MONTEVIDEO}

Las iniciativas de Segovia y el musicólogo Francisco Curt Lange finalmente cristalizaron en una invitación, fechada el 24 de mayo de 1940, del Servicio Oficial de Difusión Radio

${ }^{1}$ Amadeo Antón Vázquez, "Regresó el adelantado", publicación sin identificar (ca. 1942), Archivo Ponce (AP, en subsecuentes referencias). Agradezco al pianista Carlos Vázquez, discípulo y heredero de Ponce, por permitirme consultar el Archivo Ponce. 
Eléctrica (SODRE), "Instituto máximo de la Difusión cultural en el Uruguay"2, mismo que cuenta, entre otros recursos, con una orquesta sinfónica (OSSODRE), "considerada como la segunda en importancia en la América Hispana"3. El 21 de agosto de 1941, Ponce sale de México; hace escalas en Guatemala, Cristóbal (Panamá), Cali, Lima, Santiago de Chile, Buenos Aires y llega el 27 a Montevideo ${ }^{4}$. Para la época el vuelo fue calificado como "vertiginoso"

Segovia, quien residía en Montevideo desde $1936^{6}$, estaba feliz de hospedar en su casa a su entrañable amigo mexicano. De inmediato, ambos se enfocan en los preparativos para estrenar el Concierto para guitarra, mismos que podremos recapitular gracias a un pequeño diario $^{7}$ del compositor. Además de otras actividades, en varias entradas del diario entre los días 8 y 22 de septiembre Ponce reporta que trabaja laboriosamente copiando las partes instrumentales. Segovia colabora también.

El concierto se llevó a cabo, el 4 de octubre, en el Estudio Auditorio del SODRE, con la participación de la orquesta sinfónica de la mencionada institución, bajo el siguiente programa: Pequeña suite en estilo antiguo, Concierto del sur (estreno mundial), Poema elegíaco y Chapultepec. Lamberto Baldi, director titular del ensamble, dirigió el Concierto para guitarra, mientras que Ponce el resto del programa ${ }^{8}$. La sala es enorme y cuenta con capacidad para tres mil personas ${ }^{9}$. En sólo cuatro ensayos, se prepara la audición.

Revivamos el primer concierto de Ponce en Montevideo, a partir de las breves anotaciones en su diario:

"4 Oct. El teatro del Sodre concurridísimo -a las 6.30 de la tarde. Ministros, diplomáticos, gente chic y, en la galería, una muchedumbre expectante. Aparezco. Nutridos aplausos. La 'Suite en estilo Antiguo' sale bien. El preludio a regular velocidad. Al terminar la Fughetta, ovación. Salgo varias veces a dar las gracias. Pequeño intermedio. Voy al palco de Paquita ${ }^{10}$ para escuchar mi 'Concerto'. Magnífica interpretación de Andrés. Ovación delirante. Tengo que acompañar a Andrés y a Baldi numerosas veces a dar las gracias. Pero el público insiste y se repite el final del 'Concerto'. Éxito rotundo. 'Chapultepec' es muy aplaudido. Gran número de personas vienen a saludarnos [...] Cenamos en casa, como todas las noches. Yo, mi 'Cafecito con un poco de leche' y pan".

\footnotetext{
${ }^{2}$ AP. Dicho organismo es conocido actualmente como Servicio Oficial de Difusión, Radiotelevisión y Espectáculos (SODRE), www.sodre.gub.uy

3 "Hoy sale el maestro Manuel Ponce a su jira por Sud América", Universal Gráfico (México, D.F.), 21 de agosto, 1941.

4 Diario de vuelo, abarca las fechas del 21 al 27 de agosto, 1941, AP; Carlos González Peña, "El caballero del aire”, El Universal (México, D.F.), 15 de enero, 1942.

5 “'Cultivemos lo auténticamente popular'; dijo el músico Ponce”, El Pueblo (Montevideo), 10 de septiembre, 1941.

${ }^{6}$ Wade 2001: 40.

7 Diario, comprende las fechas del 8 de septiembre al 9 de noviembre, 1941, AP.

${ }^{8}$ Programa de concierto, 4 de octubre, 1941, AP.

${ }^{9}$ Salgado 2001: 61. Diario, 20 de septiembre, 1941

10 Paquita Madriguera, esposa de Andrés Segovia.
} 
Cuantiosas reseñas reconocen a Ponce como uno de los más sobresalientes compositores americanos de trascendencia internacional ${ }^{11}$. Destacan que su música "es magníficamente construida, inspirada y de extraordinaria calidad”. ${ }^{12}$ La publicación de El Tiempo resume: "El éxito alcanzado ayer por el maestro Manuel Ponce, perdurará por mucho tiempo en estas tierras del Plata"13.

Naturalmente que el centro de atención fue el estreno mundial del Concierto para guitarra, sobre el que nos permitimos reproducir algunas reseñas:

El Tiempo: "Esta obra tuvo un éxito sin precedentes [...] da amplio margen a la guitarra para su natural lucimiento [...] Una ovación frenética saludó a los artistas, obligándolos a repetir el tercer tiempo"14.

El Plata: "Es ésta una obra de belleza muy espontánea [...] se observa también una admirable conexión rítmica, sobre cuya base hace muchas veces Manuel Ponce, y con riqueza de sentimientos, cantar pequeñas frases melódicas de oportunos y felices contrastes" 15 .

La Mañana: “Ante el 'Concierto del Sur' cabe señalarse, ante todo, un encomiable afán de limpidez y claridad, demostrado en el manejo acertadísimo de la orquesta reducida ${ }^{16}$, y subordinada siempre a las virtudes expresivas del instrumento solista [...] Andrés Segovia nos brindó [...] una versión acreedora de los mejores elogios [...] Lamberto Baldi [...] tuvo una comprensión muy acertada del hecho artístico. También debe señalarse la admirable limpieza y ajuste logrado por la OSSODRE"17.

El segundo programa tuvo lugar el 11 de octubre en la misma sala y con la misma orquesta bajo la dirección del propio compositor. El programa incluyó: Estampas nocturnas, Concierto para piano y Ferial, esta última composición recién estrenada en México en $1940^{18}$. Un atractivo especial de la velada fue la participación como solista de la pianista española Paquita Madriguera, quien reaparecía después de un retiro voluntario de los escenarios de veinte años ${ }^{19}$.

\section{Retomemos el diario.}

“Sábado 11 [...] A las 6:30, al Sodre. Las 'Estampas' salen bien. Estallan los aplausos. Llega su turno a Paquita. El Concerto camina sin tropiezo. Gran ovación. Por último el

11 "Diéronse a conocer en el SODRE algunas obras de Manuel Ponce", El Diario (Montevideo), 5 de octubre, 1941.

12 "Magnífico concierto fue el de ayer", El Debate (Montevideo), 5 de octubre, 1941.

${ }^{13}$ C. D., "Espléndida fiesta de la música fue la de ayer en el S.O.D.R.E.: Recital Ponce-Baldi-Segovia", El Tiempo (Montevideo), 5 de octubre, 1941.

14 Ibid.

15 Alberto Soriano, "El primer concierto del maestro Manuel Ponce", El Plata (Montevideo), 5 de octubre, 1941.

${ }^{16}$ Flauta, oboe, clarinete, fagot, pandereta, timbales y cuerdas.

${ }^{17}$ R. E. L., "En el SODRE se escucharon ayer, obras de Manuel Ponce", La Mañana (Montevideo), 5 de octubre, 1941.

18 Barrón 2004: 38.

${ }^{19}$ Diario, 8 de octubre de 1941; "P. Madriguera actuará en el concierto de M. Ponce", El Plata (Montevideo), 7 de octubre, 1941. 
'Ferial' entusiasma al público y termina el 20 concierto brillantemente. Desde el Sodre fuimos al 'Rancho' del Dr. Rubino, en uno de los barrios de Montevideo”.

A diferencia del primer concierto, el segundo es seguido de una concurrida celebración. Repasemos algunas reseñas:

El Plata: "Debemos hoy expresar nuevamente que muy difícilmente se ha de encontrar en la América Latina, otro compositor que logre estructurar sus creaciones con el equilibrio y la precisión que admiramos en las producciones que Manuel Ponce nos ha hecho escuchar"20.

El Pueblo: “'Estampas Nocturnas' [...] cuatro piezas de fina inspiración y composición segura [...] Concierto para piano [...] realización juvenil de la cual al extremo de treinta años ha dicho Adolfo Salazar, que continúa siendo una obra viva, elocuente, de perfecta escritura [...] [Paquita] es una intérprete de exquisita inteligencia, dueña de un mecanismo desarrollado, de una exquisita musicalidad, de un sonido puro"21. La Mañana: El “Ferial' es, pues, una obra interesante desde todo punto de vista: por su marcado americanismo; por el justo y mesurado empleo del folklore mejicano, por la cantidad de aportaciones personales del autor, y por la limpidez de su orquestación" 22 .

\section{GÉNESIS DE UN CONCIERTO}

Con el fin de enriquecer el repertorio de su instrumento, Segovia solicitó la colaboración de importantes compositores. Uno de los primeros en responder fue Ponce, quien, según aseveración del propio guitarrista, fue el más prolífico de todos. Desde que se conocieron en 1923, iniciaron una gran amistad y colaboración, tal como se puede apreciar en las cartas del guitarrista ${ }^{23}$. Este último señala que ya desde la primavera de 1926 , Ponce gestaba los temas principales del Concierto para guitarra ${ }^{24}$. El intérprete explica que los "azares" de su vida errante lo mantuvieron alejado del compositor por largos años, impidiendo la conclusión del proyecto. Comenta que en México tocó el Concierto de Castelnuovo-Tedesco con la participación de Ponce como director. Considera que este fue un factor importante para que Ponce finalmente concretara, en 1940, su propio concierto ${ }^{25}$. Es probable que durante muchos años el autor no le diera prioridad al Concierto para guitarra debido a su pesada carga de trabajo.

20 Alberto Soriano, "El segundo concierto de Manuel Ponce", El Plata (Montevideo), 12 de octubre, 1941.

21 "En el concierto de ayer reapareció con éxito Paquita Madriguera", El Pueblo (Montevideo), 12 de octubre, 1941.

22 R. E. L., "Manuel Ponce dirigió ayer su segundo concierto", La Mañana (Montevideo), 12 de octubre, 1941.

${ }^{23}$ Cf. Segovia 1989.

${ }^{24}$ Andrés Segovia, "La aportación de Ponce", Excelsior (México, D.F.), 24 de abril, 1949, tercera sección, p. 6; Segovia 1948: 15.

25 Ibid. 


\section{BUENOS AIRES}

Segovia y Ponce llegan a Buenos Aires el 13 de octubre ${ }^{26}$ y comienzan los preparativos para el concierto que tendrá lugar el día 20 del mismo mes en el Teatro Nacional de Comedia con la participación de la orquesta de la Asociación del Profesorado Orquestal27 bajo la organización de la Asociación Wagneriana. Surgen problemas con el patrocinio de la orquesta. Ponce contribuye para franquear el contratiempo erogando fondos de su propia bolsa $^{28}$. En tres ensayos ${ }^{29}$ prepara el programa que contiene: Chapultepec, Concierto del sur, Poema elegiaco y Ferial.

El día del evento el compositor escribe en su diario:

"20 [oct.] Ensayo por la mañana en el Teatro N. de Comedia. Todo marchó bien, gracias a Dios. Por la noche, a pesar de que sopla un viento helado, el teatro está concurridísimo. Parece que todo lo que cuenta en Buenos Aires, en materia de arte está presente. A mi salida, aplausos. Después del 'Chapultepec', me llaman 3 veces. Hago que la orquesta se ponga en pie. Al terminar el primer tiempo del 'Concerto del Sur', estalla la ovación. Dejo que Andrés reciba todos los aplausos. Sigue el 'Andante'. Podría haberse escuchado una mosca volando. Otra ovación. Andrés me obliga a dar las gracias. Allegro Final -entusiasmo delirante. Saludamos y Andrés sale 4 veces solo y 4 conmigo a dar las gracias. Tanto el 'Poema elegiaco' como 'Ferial' son muy aplaudidos. Salgo 3 veces, pero dando tiempo. Y comienza el desfile. Uno de los primeros que me felicitan es Kleiber. Lista interminable: López Buchardo, Mastroggiani, Talamón, Fontova, Juan José Castro, Copland, la hija del Mtro. Williams, en nombre de éste, y una multitud de pianistas, (Uninsky, Mailuzinski [?], Gil Marchex, Lía Cimaglia, las Sandoval, etc. etc.) violinistas, cantantes (Conchita Badía) y muchísimas personas más. Al ‘Tropezón', restaurant, con Miguel del Pino, su mujer, Fontova y otros".

${ }^{26}$ Diario; Carlos González Peña, "El caballero del aire”, El Universal (México, D.F.), 15 de enero, 1942.

27 "Un concierto de obras de Manuel M. Ponce se realizó anoche", La Prensa (Buenos Aires), 21 de octubre, 1941. Sólo en este artículo aparece el nombre de la orquesta ya que ni en el programa de mano del concierto lo consignan.

28 Diversos diarios de Buenos Aires señalan que el concierto es auspiciado por la Asociación Wagneriana. Sólo un periódico menciona que el evento será patrocinado por la mencionada asociación así como por "la Organización de Conciertos Iriberri ("Se presentará mañana por la noche el ilustre compositor mexicano Manuel Ponce”, Noticias Gráficas [Buenos Aires], 19 de octubre, 1941). El programa de mano tiene la leyenda "Concierto Sinfónico Extraordinario" y lleva efectivamente el nombre de la Asociación Wagneriana, señalando que el teatro fue "cedido por la Comisión Nacional de Cultura". Asimismo, contiene diversos anuncios publicitarios de negocios musicales, entre ellos uno de la Casa Iriberri. El artículo de Carlos González Peña, "Ponce en los países del Plata", El Universal (México, D.F.), 27 de noviembre, 1941, consigna: "Todas [las obras] las dirigió Ponce, secundado por un grupo de sesenta músicos que logró reunir con sacrificios y de su propio peculio; pues la Asociación Wagneriana de Buenos Aires, organizadora de aquel acto musical, sólo contribuyó con la pequeña orquesta de dieciocho filarmónicos que debía acompañar a Segovia”. En su diario, en la entrada del día 14 de octubre, el compositor anota: "Dificultades con 'Radio Mundo' que había ofrecido la orquesta y una cantidad por transmitir el 'Concerto' de Andrés. Por fin, decidimos hacer por nuestra cuenta los gastos del Concierto, aceptando la ayuda de la Sociedad Wagneriana”. Cabe señalar que González Peña, amigo íntimo de Ponce, escribió éste y otros artículos sobre la gira a Sudamérica seguramente con información proporcionada por el propio compositor.

${ }^{29}$ Carlos González Peña, "Ponce en los países del Plata”, El Universal (México, D.F.), 27 de noviembre, 1941. El diario del compositor sólo reporta ensayos los días 15, 16 y 20 de octubre. 
El alto nivel cultural de la capital argentina así como los contratiempos mencionados hacían de este concierto la más dura prueba de la gira. Afortunadamente, los viejos amigos salen victoriosos. Ponce recordaría este concierto como uno de los momentos más felices de su vida ${ }^{30}$, junto con reportar que más de doscientas personas vinieron a felicitarlo ${ }^{31}$.

\section{RESENAS:}

El Mundo: "El ‘Concierto del Sur' [...] hispánico en su espíritu, moderno en su factura, y excepcionalmente fino y elegante en su estética [...] Insuperablemente interpretada su parte solista por Andrés Segovia, y llevada la orquesta con sumo tacto por el autor, manteniendo el equilibrio de los ingeniosos diálogos instrumentales, gustó sin reservas a la sala" 32 .

Chapultepec [...] une sabiamente a la inspiración, técnica personal y orquestación brillante y seductora [...] Poema elegíaco, página de dramático sentido espiritual [...] Es sin duda Ferial, una obra que por todos los valores que encierra, un monumento del arte de América"33.

La Nación: 'El 'Concierto del Sur' [...] es, desde luego, la obra más interesante de la audición, de una calidad poco frecuente, tanto por la riqueza y variedad de los recursos sonoros como por la gracia y profundidad de la inspiración y la habilidad técnica de que hace gala el compositor [...] el conjunto orquestal no se limita al papel de simple acompañamiento, más o menos adornado, sino que dialoga activamente entre sí, o con el instrumento principal [...] [sobre Segovia] fraseo diáfano y expresivo, extremada variedad de matices, virtuosismo técnico absoluto y, sobre todo, una madurez espiritual y perfecta identificación con el espíritu de la obra [...] Manuel M. Ponce, que se reveló como un director de orquesta sobrio y eficiente, fue muy aplaudido por el auditorio que asistió a la audición" 34 .

El estreno del Concierto del sur da inicio a una serie de éxitos. En los siguientes años, Segovia lo interpreta en diversas ciudades de América: en 1944 y 1947 en el Palacio de las Bellas Artes de la Ciudad de México con Erich Kleiber y Carlos Chávez como directores, respectivamente; en 1946, en el Carnegie Hall de Nueva York. Sobre esta última audición, el compositor y crítico musical del New York Herald-Tribune, Virgil Thomson, escribió:

"El Concierto del Sur, de Ponce, fue una delicia. Escrito a la manera andaluza, es rico en variedad expresiva e interés musical. Además, el acompañamiento orquestal amplifica el de por sí extenso rango de timbres de la guitarra en la manera más atractiva imaginable $[\ldots]$ El concierto de Ponce tuvo hondura sin pérdida alguna de la distintiva

${ }^{30}$ F. Gómez Hidalgo, "Creadores de México. El maestro Ponce”, Estampa (México, D.F.), 2 de febrero, 1943.

31 Carlos González Peña, "Ponce en los Países del Plata", El Universal (México. D.F.), 27 de noviembre, 1941 .

${ }^{32}$ L. A. G. "Aplaudiéronse obras de Ponce", El Mundo (Buenos Aires), 22 de octubre, 1941.

33 "Manuel Ponce: presentación del gran compositor mejicano", publicación sin identificar, Buenos Aires, $c a$. octubre, 1941, AP.

34 "Un concierto sinfónico de obras de M. Ponce se realizó en el T. N. de comedia", La Nación (Buenos Aires), 21 de octubre, 1941. 
intensidad expresiva de la guitarra. Su Concierto del Sur es una obra completamente deliciosa y una contribución excepcional al repertorio"35.

Por su parte, Segovia declara sobre el aludido opus: "Es la joya más preciada de mi repertorio" 36 . En otra publicación, señala que es "considerada hoy como una de las joyas representativas de la literatura guitarrística contemporánea"37.

\section{SANTIAGO DE CHILE}

El 7 de noviembre, Ponce deja Buenos Aires y llega a Santiago ${ }^{38}$ para dirigir un concierto con la Orquesta Sinfónica de Chile por invitación del Instituto de Extensión Musical ${ }^{39}$. Este programa es el tercero de la serie denominada Festivales Panamericanos. Entre los distinguidos directores de tan importantes conciertos con la Sinfónica de Chile se anuncia la participación de Oscar Lorenzo Fernandez (Brasil), Guillermo Espinosa (Bogotá), Aaron Copland (Estados Unidos) y Carlos Chávez (México) ${ }^{40}$.

El evento se fijó para el 25 de noviembre. Sin embargo, se postergó para el 2 de diciembre debido al luto nacional por la muerte del entonces Presidente de Chile, el profesor y abogado Pedro Avelino Aguirre Cerda, acaecida en Santiago precisamente el 25 de noviembre. ${ }^{41}$ El concierto se llevó a cabo en el Teatro Municipal con las siguientes obras: Pequeña Suite en estilo antiguo, Chapultepec, Poema elegíaco y Ferial.

En carta fechada el 3 de diciembre, Ponce escribe a su esposa, Clema ${ }^{42}$ :

“¡Por fin! Ayer, a las 7 de la noche se efectuó con éxito clamoroso mi concierto [...] La orquesta tocó muy bien. Muchísimas personas vinieron a saludarme: Allende, los Soro, Cotapos, Urrutia [...] todos los músicos e intelectuales chilenos [...] Gracias a Dios y a la Santísima Virgen ayer salió todo bien y todo el mundo me felicitó por mis obras y por mi dirección”.

\section{Reseñas:}

"La Nación: La música sinfónica de Ponce, acusa, en su totalidad, el alma de un artista bondadoso, de un místico, de un ser limpio de espíritu y de pensamiento, de un hombre sin problemas ni sombras en el corazón [...] El boceto sinfónico denominado 'Chapultepec', el 'Poema elegíaco' y el 'Divertimento', 'Ferial' -que el artista dirigió

35 Virgil Thomson, "Guitar with orchestra", New York Herald Tribune (Nueva York), 14 de enero, 1946. Traducción de Jorge Barrón.

${ }^{36}$ Revuelta, "Con sus propias palabras. Andrés Segovia", datos de publicación sin identificar, AP.

${ }^{37}$ R. Cárdenas, "Ecos de la voz popular", Jueves de Excelsior (México, D.F.), 4 de marzo, 1948.

38 Diario, 7 de noviembre, 1941.

39 "El maestro Ponce dirigirá la O. Sinfónica Nacional”, Diario Ilustrado (Santiago de Chile), 18 de noviembre, 1941.

40 "Activo intercambio musical americano", El Mundo (Santiago de Chile), 4 de noviembre, 1941. De los directores anunciados solamente Oscar Lorenzo Fernandez y Aaron Copland dirigieron la Orquesta Sinfónica de Chile durante la década de los 40’ (n. del e.).

${ }^{41}$ Wikipedia, Pedro Aguirre Cerda, página consultada el 31 de mayo,2010, http:/ / es.wikipedia. org/wiki/Pedro_Aguirre_Cerda.

42 Cartas de Ponce a Clema enviadas desde Santiago de Chile. Las fotocopias pertenecen a Emilio Díaz, a quien agradezco por permitirme consultar dichos documentos. 
con sobrada precisión y cuadratura- son trozos que contienen bellas evocaciones de la música azteca $[\ldots]$ estos poemas sinfónicos, cuyas cantinelas hablan, con más fuerza que una lección de historia, de una tradición continental enaltecedora y ejemplar como ninguna" 43 .

El Mercurio: "Dirigidas por el propio compositor, tuvimos oportunidad de conocer algunas obras de la producción sinfónica de Ponce, y de apreciar sus valiosas características, que lo sitúan en un rango destacado dentro de la música americana [...] 'Chapultepec' $[\ldots]$ a nuestro juicio la obra fundamental en este programa. Hay en esta partitura gran unidad, fluidez, y una vena imaginativa fresca y espontánea $[. .$.$] La orquestación es$ finamente colorista y de una claridad y equilibrio que denotan una feliz asimilación de los maestros franceses entre los cuales convivió largos años" 44 .

\section{OTRAS ACTIVIDADES}

Además de los conciertos, Ponce realizó múltiples actividades profesionales y sociales. Presentó tres conferencias: una en Montevideo y dos en Buenos Aires. En Chile, participó como jurado, junto con Copland y otros compositores, del Concurso de música de cámara y folklórica chilena. En su carácter de Secretario de Relaciones Exteriores del Sindicato de Autores, Compositores y Editores de Música de México, realizó gestiones de intercambio con la Asociación General de Autores del Uruguay y la Sociedad Argentina de Autores y Compositores. Por su parte, la Sociedad Argentina de Música de Cámara le entregó un diploma como socio honorario y la Sociedad Guitarrística de Buenos Aires lo recibió en su sede.

Ponce tenía una ocupada agenda social con actividades casi todos los días: banquetes, paseos, conciertos, encuentros con personalidades y asociaciones. El compositor fue objeto de homenajes y recepciones. Destacamos, entre otros, los ofrecidos por el Instituto Interamericano de Musicología (Montevideo), las embajadas de México (Argentina) y de Estados Unidos (Uruguay), diversas sociedades de compositores, artistas, intelectuales y periodistas.

\section{RETORNO}

En cartas a su esposa ${ }^{45}$, Ponce menciona la posibilidad de realizar conciertos en Lima y Bogotá. Sin embargo, ninguno de éstos se concretó. Para el primero, tendría que esperar hasta enero a que terminaran los conciertos que Chávez y Kleiber darían en Lima ${ }^{46}$. Respecto al segundo, reporta: "Ya no iré a Bogotá, porque terminó la temporada de conciertos" 47.

El 16 de diciembre a las siete de la mañana deja Santiago y llega a Lima ese mismo día a las seis de la $\operatorname{tarde}^{48}$. Aunque desea continuar su viaje de regreso lo antes posible, logra

43 A. A., "El tercer festival panamericano, dirigido por Manuel Ponce", La Nación (Santiago de Chile), 3 de diciembre, 1941.

${ }^{44}$ C. H. S. [Carlos Humeres Solar], "Concierto del maestro Manuel Ponce", El Mercurio (Santiago de Chile), 3 de diciembre, 1941.

45 Noviembre 21 y 26; diciembre 3, 15, 16 y 18.

46 Carta de Ponce a Clema, Santiago de Chile, 26 de noviembre, 1941.

47 Carta de Ponce a Clema, Santiago de Chile, 15 de diciembre, 1941.

48 Agenda del compositor, incluye las fechas del 12 de noviembre al 14 de enero de 1942, AP. 
conseguir boleto hasta el 24 de diciembre; pasa por Cali, Panamá, Guatemala y aterriza en la Ciudad de México el 2649. Su esposa y hermanas lo esperaban en Aguascalientes. El 28, viaja por tren para pasar una semana con su familia en plena época navideña ${ }^{50}$. En la ciudad de México a principios de 1942, artistas, diplomáticos, intelectuales y amigos celebran la gira de Ponce con elegantes banquetes en el Hotel Reforma, en la residencia del ministro de Polonia y en el Hotel Ontario ${ }^{51}$.

La gira a Sudamérica constituye un triunfo memorable en la carrera de Ponce, quien fue pionero en llevar música sinfónica mexicana a esos países. La cantidad de prensa generada por su gira, especialmente en Montevideo y Buenos Aires, es la más copiosa de su vida. Desde su estreno, el Concierto del sur quedó firmemente establecido en la literatura guitarrística mundial. De hecho, es la obra orquestal más célebre del compositor y también la que cuenta con un mayor número de grabaciones por distinguidos intérpretes ${ }^{52}$.

\section{BIBLIOGRAFÍA}

\section{Archivos}

Archivo Ponce (abreviado AP)

\section{Periódicos}

\section{Buenos Aires}

"Se presentará mañana por la noche el ilustre compositor mexicano Manuel Ponce", Noticias Gráficas, 19 de octubre,1941.

"Un concierto de obras de Manuel M. Ponce se realizó anoche”, La Prensa, 21 de octubre, 1941.

"Un concierto sinfónico de obras de M. Ponce se realizó en el T. N. de comedia”. La Nación, 21 de octubre, 1941.

L. A. G. “Aplaudiéronse obras de Ponce”, El Mundo, 22 de octubre, 1941.

"Manuel Ponce: presentación del gran compositor mejicano", Publicación sin identificar, ca. octubre, 1941, AP.

Ciudad de México

"Hoy sale el maestro Manuel Ponce a su jira por Sud América”, Universal Gráfico, 21 de agosto, 1941.

González Peña, Carlos. "Ponce en los países del Plata”, El Universal, 27 de noviembre, 1941.

González Peña, Carlos. “El caballero del aire”, El Universal, 15 de enero, 1942.

49 Carlos González Peña, "El caballero del aire”, El Universal (México, D.F.), 15 de enero, 1942.

50 Ibid.

51 "El homenaje al maestro Manuel M. Ponce”, El Universal (México, D.F.), 12 de febrero, 1942; "Homenaje a un célebre músico", Excelsior (México, D.F.), 12 de febrero, 1942; "Una comida al maestro Manuel M. Ponce”, publicación sin identificar, ca. 28 de febrero, 1942, AP; "Homenaje del Ateneo a un Maestro", Excelsior (México, D.F.), 18 de marzo, 1942.

52 Para un listado de grabaciones, véase Barrón 2004: 34. 
"El homenaje al maestro Manuel M. Ponce", El Universal, 12 de febrero, 1942.

"Homenaje a un célebre músico", Excelsior, 12 de febrero, 1942.

"Una comida al maestro Manuel M. Ponce", publicación sin identificar, ca. 28 de febrero, 1942, AP.

"Homenaje del Ateneo a un Maestro", Excelsior, 18 de marzo, 1942.

Vázquez, Amadeo Antón. "Regresó el adelantado", publicación sin identificar, ca. 1942, AP.

Gómez Hidalgo, F. “Creadores de México. El maestro Ponce”, Estampa (México, D.F.), 2 de febrero, 1943.

Cárdenas, R. "Ecos de la voz popular”, Jueves de Excelsior (México, D.F.), 4 de marzo, 1948.

Segovia, Andrés. "La aportación de Ponce”, Excelsior, 24 de abril, 1949, tercera sección, 6.

Revuelta. "Con sus propias palabras. Andrés Segovia”, datos de publicación sin identificar, AP.

Montevideo

“'Cultivemos lo auténticamente popular'; dijo el músico Ponce”, El Pueblo, 10 de septiembre, 1941.

C. D. "Espléndida fiesta de la música fue la de ayer en el S.O.D.R.E.: Recital Ponce-Baldi-Segovia", El Tiempo, 5 de octubre, 1941.

R. E. L. "En el SODRE se escucharon ayer, obras de Manuel Ponce", La Mañana, 5 de octubre, 1941.

Soriano, Alberto. "El primer concierto del maestro Manuel Ponce", El Plata, 5 de octubre, 1941.

"Diéronse a conocer en el SODRE algunas obras de Manuel Ponce", ElDiario, 5 de octubre, 1941.

“Magnífico concierto fue el de ayer”, El Debate, 5 de octubre, 1941.

"P. Madriguera actuará en el concierto de M. Ponce”, El Plata, 7 de octubre, 1941.

R.E.L. "Manuel Ponce dirigió ayer su segundo concierto", La Mañana, 12 de octubre, 1941.

Soriano, Alberto. "El segundo concierto de Manuel Ponce", El Plata, 12 de octubre, 1941.

"En el concierto de ayer reapareció con éxito Paquita Madriguera", El Pueblo, 12 de octubre, 1941.

NuEva YoRK

Thomson, Virgil. "Guitar with orchestra”, New York Herald Tribune, 14 de enero, 1946.

Santiago de Chile

“Activo intercambio musical americano", El Mundo, 4 de noviembre, 1941.

“El maestro Ponce dirigirá la O. Sinfónica Nacional”, Diario Ilustrado, 18 de noviembre, 1941.

A. A. "El tercer festival panamericano, dirigido por Manuel Ponce", La Nación, 3 de diciembre, 1941.

C. H. S. [Carlos Humeres Solar]. "Concierto del maestro Manuel Ponce”, El Mercurio, 3 de diciembre, 1941. 
Articulos y libros

Barrón Corvera, Jorge

2004 Manuel M. Ponce: A Bio-Bibliography. Westport, Connecticut: Greenwood Publishing Group; Praeger Publishers.

Gómez Hidalgo, F

1943 “Creadores de México. El maestro Ponce”, Estampa (México, D.F.), 2 de febrero.

Salgado, Susana

2001 "Montevideo", The New Grove Dictionary of Music and Musicians. Segunda edición. Editado por Stanley Sadie. Londres: Macmillan Publishers Ltd., p. 6.

Segovia, Andrés

1948 "Manuel M. Ponce, notas y recuerdos", Guitar Review, II/7, pp. 15-16.

1949 “La aportación de Ponce”, Excelsior (México, D.F.), 24 de abril, 1949, tercera sección, p. 6.

1989 The Segovia-Ponce Letters. Edición de Miguel Alcázar. Traducción de Peter Segal. Columbus, Ohio: Editions Orphée.

WADE, GRAHAM

2001 "Segovia, Andrés", The New Grove Dictionary of Music and Musicians. Segunda edición. Editado por Stanley Sadie. Londres: Macmillan Publishers Ltd., p. 61. 